\title{
An Approach for a Negotiation Model Inspired on Social Networks
}

\author{
João Carneiro $^{1}$, Goreti Marreiros ${ }^{1}$, and Paulo Novais ${ }^{2}$ \\ ${ }^{1}$ GECAD - Knowledge Engineering and Decision Support Group, Institute of Engineering - \\ Polytechnic of Porto, Porto, Portugal \\ \{jomrc, mgt\} @isep.ipp.pt \\ ${ }^{2}$ CCTC - Computer Science and Technology Center, at University of Minho, \\ Braga, Portugal \\ pjon@di.uminho.pt
}

\begin{abstract}
Supporting group decision-making in ubiquitous contexts is a complex task that needs to deal with a large amount of factors to be successful. Here we propose an approach for a negotiation model to support the group decisionmaking process specially designed for ubiquitous contexts. We propose a new look into this problematic, considering and defining strategies to deal with important points such as the type of attributes in the multi-criteria problem and agents' reasoning. Our model uses a social networking logic due to the type of communication employed by the agents as well as to the type of relationships they build as the interactions occur. Our approach intends to support the ubiquitous group decision-making process in a similar way to the real process, which simultaneously preserves the amount and quality of intelligence generated in face-to-face meetings and is adapted to be used in a ubiquitous context.
\end{abstract}

Keywords: Group Decision Support Systems, Ubiquitous Computing, Automatic Negotiation, Social Networks, Multi-Agent Systems

\section{Introduction}

Many existing Group Decision Support Systems (GDSS) prototypes use automatic negotiation models as a strategy to support the decision [1-4]. Argumentation-based negotiation models are one of the most used and best suited automatic negotiation technics to support the decision-making [5, 6]. It is consensual that the possibility of justifying a request using an argument facilitates reaching an agreement or solution $[6,7]$. Albeit all the recognized advantages in the use of argumentation models in decision-making, and the time necessary to study argumentative models in the area of computer science is traced back to a few decades, the truth is that such models have not yet been embraced by organizations. The existing models are barely adaptable to the business world reality, have difficulty in reflecting the decision-making natural process, and create a certain discomfort in their use by decision-makers. It is also important to note that the actual evaluation of the argumentation models is not the one 
an organization would want to use. The fact an argumentation model gives a solution in lesser rounds or in lesser seconds than another, are not the most relevant points for whom is concerned about using a mechanism to potentiate the decision quality. Maybe because of that, business intelligence techniques have a much higher growth than GDSS.

In literature there are various negotiation models adapted to group decision-making [8-12]. However, the existing models are limited to very specific contexts and do not support the decision-maker in the smarter way. Looking for studies on argumentationbased negotiation models adapted to group decision support systems, the results are practically inexistent. The few existing results are old [11, 13] and if some seemed promising in the way they could be adapted to this area $[14,15]$, the works that came next followed most of the times another path (even with some of them remaining within decision support). Forgetting negotiation models for a moment, we find that even the existing argumentation approaches are not oriented to problems that include multiple agents simultaneously. It is even possible to verify that in the most recent argumentation studies, authors with more than a decade of work, point the inclusion of multiple agents as a future expansion for their work [16, 17]. When agents have "one-to-one" communication the process is simple. However, things become more difficult when an agent receives messages from multiple agents. Another important issue is how most authors test their argumentation models, the majority opt for the "seller-buyer", example [5, 6, 18-21], which has a type of dialogue much oriented to that kind of problem.

Defining a type of adaptable dialogue for use in an argumentation-based negotiation model which has the objective to support group decision-making is a complex task. Walton [22] believes that dialogues should be classified based in their primary objective, and presents six major dialogue classes for that: inquisition, persuasion, negotiation, deliberation, demand for information and eristic. However, what is the most adaptable dialogue for a group of people, employees of the same company, whose common objective is to solve a certain problem, but at the same time satisfy their own objectives? Maybe a mix of several types of dialogue could be the solution, or the creation of a new class. This makes it very complex to adapt an argumentation theory to this scenario.

We believe that part of the failure of group decision support systems developed until today is due to the perspective used to analyse the problem and how they have been evaluated.

Here we propose a theoretical negotiation model that intends to support the ubiquitous group decision making process similarly to a real process, which simultaneously preserves the amount and quality of intelligence generated in face-to-face meetings and is adapted to be used in a ubiquitous context. To achieve this, a model that uses a social networking logic is proposed. The model is based on the agents' type of communication and type of relationships that they build as the interactions occur.

With the inclusion of the work we have been developing [23, 24] in the model here presented we believe it will be possible to enhance the decision quality.

The rest of the paper is organized as follows: in the next section is presented our approach, where the theoretical ideas for the negotiation model are described, and the 
attribute types' definition and the agents' reasoning are presented. Finally, some conclusions are taken in section 3 , along with the work to be done hereafter.

\section{Proposed Model}

The model here proposed is theoretical and results from the study conducted by the authors in the last years in this area. It seems clear that despite decades of study in the GDSS area they have had acceptance difficulties by the firms. On the other hand, the business intelligence techniques that came from decision support systems have had a great acceptance in the last years. We believe our approach can eventually be a solution to the problems that are leading to the difficulty in accepting the GDSS. Thus, in this section we present our theoretical negotiation model, as well as we identify some of the points we consider as problematic, proposing solutions and explaining how our approach can solve those problems. Besides its clear objective, this proposal aims to be an incentive to reflection for the researchers working in this area.

Much of the existing literature that uses agents for negotiation purposes [25-27] mainly considers scenarios where the agents are fully competitive, in which each agent seeks to achieve its own goals $[28,29]$ or fully collaborative, where all seek to find a solution that is satisfactory to all [30-32]. In the case of a GDSS that aims to support an organization's decision group to make decisions, this issue should be viewed with other sensitivity. Considering a system will have agents, where each agent represents a decision-maker, they should be a mix of competition and collaboration. We could consider that as all the agents are part of the same organization, they should be collaborative in that all seek to achieve the best possible decision for the firm. However, for human nature reasons, that would lose certain existing advantages in the context of meeting. Although "all wear the same sweater", in a real context the decision-makers also seek to achieve their own goals. This happens for several reasons, but in this situation we are interested in highlighting that this happens for conviction reasons. The decision-maker considers in his logic that his preferred alternative is the best solution to solve the problem and will defend his alternative until arguments that make him consider a more benefic alternative are used. It is this behavior that enriches the meetings, introduces new knowledge and allows higher quality decisions to be attained. This is the behavior we intend to include in our negotiation model and that we consider to be important to introduce in this kind of systems.

The negotiation model here proposed is inspired by the communication logic used in social networks. The main idea follows two main types of communication: Public Communication (PC) in the form of public posts, and Private Communication (PrC) in the form of private chat. The visual idea of the communication form is much alike to the one used for instance in Facebook®. The fact of considering the way of communication used in social networks a good approach to serve as inspiration for this work topic is related to three main factors: the agents communicate in a context similar to the one practiced by the decision-makers in face-to-face meetings, the environment and the agents communication/interaction is easily understood by the partici- 
pants (decision-makers), and the possibility to use the techniques already developed to study the relationships (in the social networks literature).

Fig. 1 represents the two different types of communication. The agent is part of a single PC but can have several PrC simultaneously.

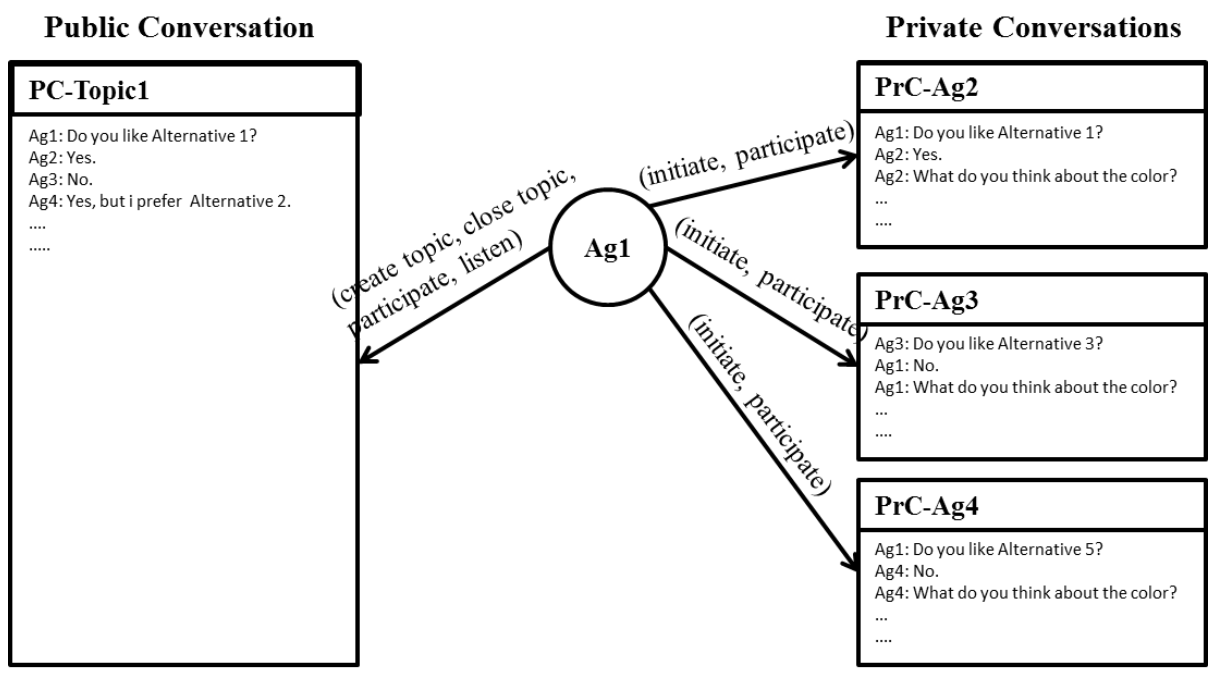

Fig. 1. The two different types of communication

A PC is an open conversation and its functioning reflects the type of dialogue practiced by the decision-makers in a real context. Sometimes public conversations or conversations between multiple agents are mentioned, but in practice what happens is that there is a group of agents that exchanges messages where each message has a single receptor. In the case of PC, messages are exchanged as happens in reality, where a group of people are seating at a table and even when a message has only one recipient it can be heard by all. This allows the agents to gather information and create relationships through the messages they listen, even when they are not directed to them. In PC agents can only address one topic at a time, i.e., there is any participant agent at a certain time when there is not an open topic that creates a topic about a certain theme. As mentioned, there can only be an open topic at a time. Any agent can propose the closure of a topic, which will be closed if no agent has anything else to say. Obviously all agents can participate in a PC and read all the messages.

PrC are all the private conversations of each participant agent, and as mentioned, an agent can keep several PrC simultaneously. At most, it can have a PrC with each of the other agents. An agent can initiate a PrC with any other agent provided it does not already exist. A PrC can stay open during the entire process without the need to be terminated. The existence of $\operatorname{PrC}$ are an advantage over the actual meetings that do not allow simultaneous private conversations during the process.

In literature (to the best of our knowledge) in the context of support for group decision-making the agents use requests and questions as a way of communication. The 
communication allows them to use strategies to persuade the other agents as well as to gather the necessary information to reason about the problem. In addition to questions and requests, in our approach we introduce the concept of statement. The statement is a way of communication that will be used by the agents to demonstrate their points of view. This means agents can share information or perform indirect persuasion through statements. For instance, Agentl can say "to me consumption is the most important attribute". For example, this action can make Agent2, which considers Agent1 as the most expert in the issue that is being discussed, to redefine the importance he gives to the consumption attribute. Another situation that may occur with this statement is that other agents can create a relationship with a certain strength (see 2.2) with Agent1 because they identify themselves with his point of view. As mentioned earlier, it is essential to give prominence to the decision process since strategies that propose solutions based on the problem's initial settings lose the process's richness existent in real meetings. Negotiation automation should continue to allow the existence of two fundamental questions: change of opinion/problem reformulation by the decision-makers when they realize/agree with the arguments presented by other interveners, and learning with the assessment of the process by the decision-makers. Statements, requests and questions can be used with and without the inclusion of arguments and can be used in PC and PrC. Counter-arguments and acceptance or rejection responses are also made through those three types. A communication is defined as follows:

$$
\text { Type(Sender, Receiver, Message, Args, CC) }
$$

Where:

- Type: Is the type of message, which can be a statement, message or question. For example, a response to a request or question is always of the type statement;

- Sender: Is the agent that communicates/sends the message;

- Receiver: Is the agent or group of agents that receive the message. In the case of PC there can be an addressed agent although all agents can read the message. In PrC there is always just one recipient;

- Message: Is the message that is of type Type;

- Args: Are the set of arguments used to justify the message;

- $C C$ : Is the conversation's identification code where the message will be read.

\subsection{Attribute Types}

Our model is specifically designed to handle with multi-criteria problems. It is not our goal to include any type of natural language mechanism in our prototype. However, we believe it is possible and essential that the agents understand what is happening in the "conversation". For that it is necessary to make a proper definition of type of attributes used.

Let us imagine that a group of decision-makers need to decide on a new car to add to the company's fleet. Considering one of the attributes as the car's consumption and that it was defined as a minimization numerical attribute, if Agent1 says "for me the 
most important decision factor is consumption" it will allow other agents to argue with Agent1 saying "accept alternative $\mathrm{C}$ because it has the lowest consumption". As it is possible to understand this strategy allows the agents to have the ability to perceive a lot of different information. Another major advantage of this approach is the easiness an agent has in generating perceptible reports for the real participant. Besides being able to present data that supports the decision (for instance, charts, tables, statistics, etc) it also allows to present the argumentation between the agents and the motive that led the agents to propose a certain decision in a perceptible way.

The types of attributes considered can be visualized in Fig. 2. Two main types of attributes can be considered:

- Objective: objective attributes are comparable with each other. This means that in the case of the car consumption, if car1 has a lower consumption than car2 and the consumption is a minimization numerical attribute, carl's consumption is invariably better than car2's consumption. The values of the objective attributes are always absolutely true. For instance, if the air conditioning attribute of an alternative is true then the possibility of that car not to have air conditioning cannot be considered. There are three types of objective attributes:

- Boolean: are used in situations where the attribute can be classified by only two values, e.g., on/off, yes/no, 0/1, true/false; in this case the most advantageous situation must be specified (true or false). However, this specification is not mandatory. The situation that offers a greater value is considered to be advantageous even if that value does not suits the problem to be solved. Considering the same car that with and without air conditioning costs exactly the same price, the fact of having air conditioning is an advantage, even assuming that for health reasons it will not be used;

- Numerical: the numerical type attributes are used to define measurable attributes, for example: consumption, height, width and distance. This type of attribute is defined as maximization or minimization attribute. However, this specification is not mandatory. For instance, we "always" want to minimize costs, but on the other hand, we always want to maximize the profits. However, we may not be interested in minimizing or maximizing an employer's height.

- Classificatory: this type of attribute is used to specify attributes with a defined and recognized classification. For instance, we can use this type of attribute to specify a car's safety. However, this classification should not be made by someone without credentials. An expert or a classification that has been published in a reference location can be used to make this classification. The classification will function as a scale.

- Subjective: subjective attributes allow agents to perceive what issues do not make sense to argue. For example, it will not make sense to argue a car is better than another because of the color. The fact an agent prefers a certain color (in a certain context) is considered by this type of attribute as a personal taste about which I cannot argue. Other examples of subjective attributes (always depends on the context) are: car design, food taste, beauty, sound quality, etc. 


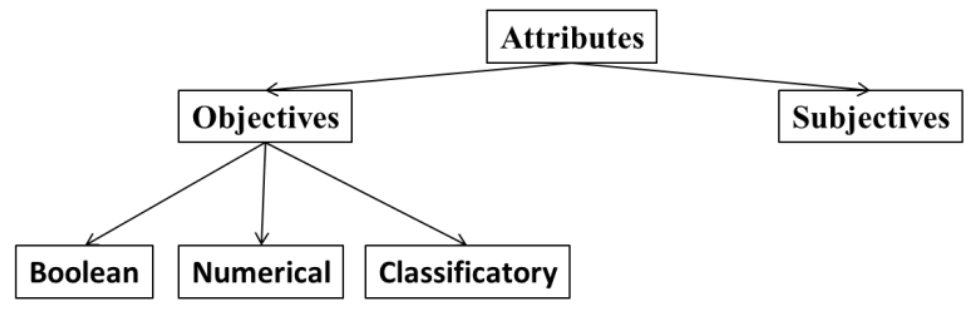

Fig. 2. Attributes' Types

We believe this proposal on the types of attributes for the multi-criterion problem is simple but effective. This way it is possible to set a lot of problems with a strategy that allows agents to understand about what they are arguing. We believe this approach makes the agents as well as the dialogues more intelligent allowing richer and perceptible outputs.

\subsection{Agents' Reasoning}

To Jennings and Wooldridge an intelligent agent is capable of flexible autonomous actions in order to meet its design objectives [33]. To them, an intelligent agent needs to be: responsive, proactive and social (for further information about these definitions see [33]). To Wooldridge what makes a rational agent is its autonomy [34]. In the last decades we have seen many examples in literature that address the topic of intelligent agents [35-37]. It is also known that there are agents that perform the same task more intelligent than others. However, if it is known that in the case of humans the reactive decision is processed by the brain in a different location of the proactive decision, in the case of agents or computational systems the proactive decision can exist but always in a simulated way.

On the subject of intelligent and rational agents, there is a relevant point that merits consideration regarding the group decision-making support systems. Let's suppose we have a system that rapidly can propose a solution to a certain problem according to the decision-makers preferences. It is obvious that this indicator is not enough to know whether the system is good or bad. The proposed solutions can always be unacceptable for the decision-makers making the system useless. However, let us consider the system can always propose acceptable solutions for the decision-makers ending up to have a great impact on a particular organization. Taking into account these data it would be hypothetically possible to say this system had quality. However, this may not be true. When someone wants to develop a negotiation model to adapt to a group decision-making support system there is an important factor that normally is forgotten. In the case of face-to-face meetings the decision-makers have time to think over the subject during the process, and often they start the meeting with certain beliefs which are then changed when they hear the others' opinion and argumentations. Sometimes our opinion changes when new knowledge is demonstrated to us or when the arguments used invalidate our logic. This fact is what makes face-to-face meetings the choice to make important decisions, and no system is still prepared to deal with 
this situation. The way models and systems are designed make this crucial part of a real meeting to be lost. This led us to think that research on negotiation models for group decision-making support systems needs to start concerning about that. It is important the agent has the capability to seek to understand why other agents have other preferences, and not only seek to achieve his goals forgetting that on the other side there may be an agent that can change of opinion without sharing the why of his initial convictions with the group.

In the approach here presented, and as already explained, it is intended that the agents communicate in public and private. Public communication is visible by all agents even if it is not directed towards a specific agent. As such, an agent will be listening to a public conversation even if he is not part of it. The agent shall gather information on the publically exchanged messages and process that information. The idea here is that the agent studies the relationships that are being created as the information is exchanged. In a real meeting, if one of the decision-makers shows his preference for a certain alternative or attribute that is also my favorite, in that instant a connection between us is created because we have that in common. Those created ties or relationships can be analyzed by social network algorithms in literature $[38,39]$. The idea is to create a new link (or update it) in a directed weighted graph (Fig. 3) every time an agent reads a public message.

By creating the graph the agent can make several analyses depending on the algorithms used. The agent can create multiple graphs on different topics where the weight of the connection is related to the graph's topic. This will allow the agent to understand, even without interacting directly with every agent, which agents are the closest in certain ideas as well as the hypothetical groups that are in agreement. New arguments can be generated from the graph analysis, for instance, it is possible to understand if there is a majority towards an alternative, among other more complex analyses.

Another topic that also will be part of the agents' reasoning and whose advantages have already been previously addressed is the capability to seek to understand the why of the other agents' preferences. If we think clearly this approaches the agents' reasoning of what happens in reality: a decision-maker seeks to understand the why of the other decision-makers' opinion. Again, this will allow to generate a richer argumentation as well as to generate more useful and elaborated reports to be analyzed by the decision-maker. The agent will have the ability to understand why by analyzing and questioning the other agents on the evaluation and importance they give to the attributes. In the example of buying a car, if an agent gives much importance to the consumption and that agent has a preferred car which is the one with the lowest consumption, another agent can deduce that this is why he chooses that alternative. This will allow him to tell the agent to switch to his preference of another car which has a slightly higher consumption but is much cheaper, arguing that the difference he will spend on fuel is insignificant.

Finally, the agents will have the ability to analyze the prediction they make on their satisfaction, that is, the prediction on their perception of the decision quality at a given moment, taking into account the outcome they are predicting to happen. For that, they will use our model on satisfaction analysis previously published by us (for fur- 
ther information read $[23,24])$. The fact they have the ability to analyze the final satisfaction of the decision-maker they represent makes them more intelligent. This allows them to know when they have to stop defending their favorite alternative to bet on another also favorite (although less) that will give them a greater final satisfaction than another they are predicting to be chosen. The model also allows to predict the group final satisfaction when their goal is a high satisfaction for all the elements. Satisfaction analysis will also be useful for blockage situations and will help the agents to better understand whether or not to accept requests from other agents.

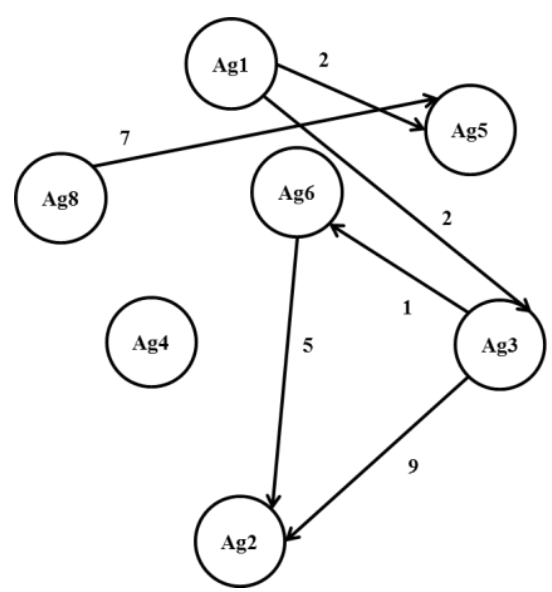

Fig. 3. Example of a directed weighted graph of relationships between agents regarding a subject

\section{Conclusions and Future Work}

The group decision support systems have been studied in the last three decades. However, after all this time, they are still facing problems in being accepted by the industry. Regardless the amount of artificial intelligence techniques applied, they still have too many limitations, especially in situations with time/space constraints. Furthermore, there are big challenges regarding the processes used to evaluate and validate these systems. The used evaluation processes allows to have good scientific results in certain cases but do not transmit enough confidence so the industry can understand all the potential of these systems.

In order to support the group decision-making in situations with time/space constraints, the GDSS evolved for the so-called Ubiquitous GDSS (UbiGDSS). They are the ultimate cleavage of GDSS. With the appearance of UbiGDSS some other problems appeared, for instance, how to: overcome the lack of human-interaction, understand the decision quality perception in the perspective of each decision-maker and overcome the communication issues.

One of the usual techniques in UbiGDSS is automated negotiation. The idea behind automated negotiation, as for instance argumentation, is allowing agents to find a 
solution through an intelligent dialogue. However, there are no specific defined dialogues for these situations, plus there are only a few argumentation-based negotiation models proposed in literature where the majority was defined before the appearance of UbiGDSS. Going deeply, we can also verify that even the argumentation theories have difficulty in adapting to this scenario.

Here we propose a theoretical negotiation model specifically planned for ubiquitous decision-making support systems. More particularly, we propose new approaches on topics such as the type of attributes in a multi-criteria problem and the agents' reasoning. In addition to these specific proposals, this topic is addressed under a new look and approach. Multiple reflections are shared, as well as analysed issues that in the opinion of authors have been the cause of the GDSS problems.

The model proposed in this paper uses a social networking logic due to the type of communication employed by the agents, as well as to the type of relationships they build as the interactions occur. Our approach intends to support the ubiquitous group decision-making process, in a similar way to a real process, which simultaneously preserves the quantity and quality of intelligence generated in face-to-face meetings and is adapted to be used in a ubiquitous context.

As for future work there are still a lot of things that need to be done. We will work on the creation of the argumentation framework, and after that we will define all the concepts behind the dialogues. At a later stage we will develop a new prototype that will include all the topics addressed here and others previously published. We believe that in the end we can draw strong conclusions on the results obtained from using this new look over automatic negotiation in group decision-making support systems.

As a final remark, we can say that there is a lot of work to do to adapt GDSS to this new Era. This is a very complex area and involves so many different other areas, but working in this field is so much exciting and can result in outstanding results.

\section{Acknowledgements}

This work is part-funded by ERDF - European Regional Development Fund through the COMPETE Programme (operational programme for competitiveness) and by National Funds through the FCT - Fundação para a Ciência e a Tecnologia (Portuguese Foundation for Science and Technology) within project FCOMP-01-0124FEDER-028980 (PTDC/EEISII/1386/2012) and SFRH/BD/89697/2012.

\section{References}

1. Herrera, F., Herrera-Viedma, E., Verdegay, J.: A rational consensus model in group decision making using linguistic assessments. Fuzzy Sets and Systems 88, 31-49 (1997)

2. Maznevski, M.L.: Understanding our differences: Performance in decision-making groups with diverse members. Human relations 47, 531-552 (1994)

3. Moreno-Jiménez, J., Aguarón, J., Escobar, M.: The core of consistency in AHP-group decision making. Group Decision and Negotiation 17, 249-265 (2008) 
4. $\mathrm{Xu}, \mathrm{Z}$ :: An automatic approach to reaching consensus in multiple attribute group decision making. Computers \& Industrial Engineering 56, 1369-1374 (2009)

5. Rahwan, I., Ramchurn, S.D., Jennings, N.R., Mcburney, P., Parsons, S., Sonenberg, L.: Argumentation-based negotiation. The Knowledge Engineering Review 18, 343-375 (2003)

6. Marey, O., Bentahar, J., Asl, E.K., Mbarki, M., Dssouli, R.: Agents' Uncertainty in Argumentation-based Negotiation: Classification and Implementation. Procedia Computer Science 32, 61-68 (2014)

7. Bonzon, E., Dimopoulos, Y., Moraitis, P.: Knowing each other in argumentation-based negotiation. In: Proceedings of the 11th International Conference on Autonomous Agents and Multiagent Systems-Volume 3, pp. 1413-1414. International Foundation for Autonomous Agents and Multiagent Systems, (2012)

8. Ito, T., Shintani, T.: Persuasion among agents: An approach to implementing a group decision support system based on multi-agent negotiation. In: International Joint Conference on Artificial Intelligence, pp. 592-599. Citeseer, (1997)

9. Kudenko, D., Bauer, M., Dengler, D.: Group decision making through mediated discussions. User Modeling 2003, pp. 238-247. Springer (2003)

10. Gordon, T.F., Karacapilidis, N.: The Zeno argumentation framework. In: Proceedings of the 6th international conference on Artificial intelligence and law, pp. 10-18. ACM, (1997)

11. Karacapilidis, N., Papadias, D.: Computer supported argumentation and collaborative decision making: the HERMES system. Information systems 26, 259-277 (2001)

12. Marreiros, G., Santos, R., Ramos, C., Neves, J.: Context aware emotional model for group decision making. (2010)

13. Karacapilidis, N., Papadias, D.: A group decision and negotiation support system for argumentation based reasoning. Learning and Reasoning with Complex Representations, pp. 188-205. Springer (1998)

14. Kraus, S., Sycara, K., Evenchik, A.: Reaching agreements through argumentation: a logical model and implementation. Artificial Intelligence 104, 1-69 (1998)

15. Sierra, C., Jennings, N.R., Noriega, P., Parsons, S.: A framework for argumentation-based negotiation. Intelligent Agents IV Agent Theories, Architectures, and Languages, pp. 177192. Springer (1998)

16. Fan, X., Toni, F.: Decision making with assumption-based argumentation. Theory and Applications of Formal Argumentation, pp. 127-142. Springer (2014)

17. Fan, X., Toni, F., Mocanu, A., Williams, M.: Dialogical two-agent decision making with assumption-based argumentation. In: Proceedings of the 2014 international conference on Autonomous agents and multi-agent systems, pp. 533-540 (2014)

18. Karunatillake, N.C., Jennings, N.R.: Is it worth arguing? Argumentation in Multi-Agent Systems, pp. 234-250. Springer (2005)

19. Ramchurn, S.D., Sierra, C., Godo, L., Jennings, N.R.: Negotiating using rewards. Artificial Intelligence 171, 805-837 (2007)

20. de Melo, C.M., Carnevale, P., Gratch, J.: The effect of expression of anger and happiness in computer agents on negotiations with humans. In: The 10th International Conference on Autonomous Agents and Multiagent Systems-Volume 3, pp. 937-944. International Foundation for Autonomous Agents and Multiagent Systems, (2011)

21. El-Sisi, A.B., Mousa, H.M.: Argumentation based negotiation in multiagent system. In: Computer Engineering \& Systems (ICCES), 2012 Seventh International Conference on, pp. 261-266. IEEE, (2012) 
22. Walton, D.: Commitment in dialogue: Basic concepts of interpersonal reasoning. SUNY press (1995)

23. Carneiro, J., Santos, R., Marreiros, G., Novais, P.: Overcoming the Lack of HumanInteraction in Ubiquitous Group Decision Support Systems. (2014)

24. Carneiro, J., Santos, R., Marreiros, G., Novais, P.: Understanding Decision Quality through Satisfaction. Highlights of Practical Applications of Heterogeneous Multi-Agent Systems. The PAAMS Collection, pp. 368-377. Springer (2014)

25. Huang, P., Sycara, K.: A computational model for online agent negotiation. In: System Sciences, 2002. HICSS. Proceedings of the 35th Annual Hawaii International Conference on, pp. 438-444. IEEE, (2002)

26. Kakas, A., Moraitis, P.: Adaptive agent negotiation via argumentation. In: Proceedings of the fifth international joint conference on Autonomous agents and multiagent systems, pp. 384-391. ACM, (2006)

27. Rahwan, I., Sonenberg, L., Jennings, N.R., McBurney, P.: Stratum: A methodology for designing heuristic agent negotiation strategies. Applied Artificial Intelligence 21, 489-527 (2007)

28. Santos, R., Marreiros, G., Ramos, C., Neves, J., Bulas-Cruz, J.: Using personality types to support argumentation. Argumentation in Multi-Agent Systems, pp. 292-304. Springer (2010)

29. Rosaci, D.: Trust measures for competitive agents. Knowledge-Based Systems 28, 38-46 (2012)

30. Yen, J., Yin, J., Ioerger, T.R., Miller, M.S., Xu, D., Volz, R.A.: Cast: Collaborative agents for simulating teamwork. In: International Joint Conference on Artificial Intelligence, pp. 1135-1144. LAWRENCE ERLBAUM ASSOCIATES LTD, (2001)

31. Reicher, S., Haslam, S.A., Hopkins, N.: Social identity and the dynamics of leadership: Leaders and followers as collaborative agents in the transformation of social reality. The Leadership Quarterly 16, 547-568 (2005)

32. Allen, J., Blaylock, N., Ferguson, G.: A problem solving model for collaborative agents. In: Proceedings of the first international joint conference on Autonomous agents and multiagent systems: part 2, pp. 774-781. ACM, (2002)

33. Wooldridge, M., Jennings, N.R.: Intelligent agents: Theory and practice. The knowledge engineering review 10, 115-152 (1995)

34. Wooldridge, M.J.: Reasoning about rational agents. MIT press (2000)

35. Müller, J.P.: The design of intelligent agents: a layered approach. Springer (1996)

36. Sycara, K., Pannu, A., Williamson, M., Zeng, D., Decker, K.: Distributed intelligent agents. IEEE Intelligent Systems 11, 36-46 (1996)

37. Jennings, N.R., Wooldridge, M.: Applications of intelligent agents. Agent technology, pp. 3-28. Springer (1998)

38. Borgatti, S.P.: Identifying sets of key players in a social network. Computational \& Mathematical Organization Theory 12, 21-34 (2006)

39. Varlamis, I., Eirinaki, M., Louta, M.: A study on social network metrics and their application in trust networks. In: Advances in Social Networks Analysis and Mining (ASONAM), 2010 International Conference on, pp. 168-175. IEEE, (2010) 